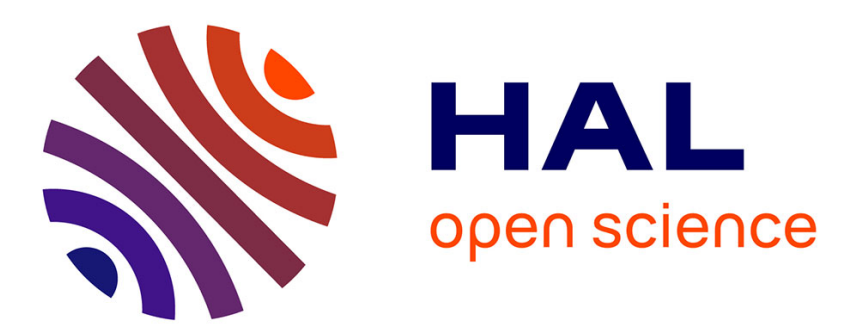

\title{
NenuFAR: Instrument description and science case
}

Philippe Zarka, Michel Tagger, L. Denis, J.N. Girard, A. Konovalenko, M.

Atemkeng, M. Arnaud, S. Azarian, M. Barsuglia, A. Bonafede, et al.

\section{To cite this version:}

Philippe Zarka, Michel Tagger, L. Denis, J.N. Girard, A. Konovalenko, et al.. NenuFAR: Instrument description and science case. 2015 International Conference on Antenna Theory and Techniques (ICATT), Apr 2015, Kharkiv, Ukraine. 10.1109/ICATT.2015.7136773 . hal-01196457

\section{HAL Id: hal-01196457 \\ https://hal.science/hal-01196457}

Submitted on 24 Sep 2020

HAL is a multi-disciplinary open access archive for the deposit and dissemination of scientific research documents, whether they are published or not. The documents may come from teaching and research institutions in France or abroad, or from public or private research centers.
L'archive ouverte pluridisciplinaire HAL, est destinée au dépôt et à la diffusion de documents scientifiques de niveau recherche, publiés ou non, émanant des établissements d'enseignement et de recherche français ou étrangers, des laboratoires publics ou privés. 


\title{
NENUFAR: INSTRUMENT DESCRIPTION AND SCIENCE CASE
}

${ }^{1}$ P. Zarka, ${ }^{2}$ M. Tagger, ${ }^{3}$ L. Denis, ${ }^{4}$ J. N. Girard, ${ }^{5}$ A. Konovalenko, ${ }^{6}$ M. Atemkeng, ${ }^{7}$ M. Arnaud, ${ }^{8}$ S. Azarian, ${ }^{9}$ M. Barsuglia, ${ }^{10}$ A. Bonafede, ${ }^{11}$ F. Boone, ${ }^{12}$ A. Bosma, ${ }^{13}$ R. Boyer, ${ }^{14}$ M. Branchesi, ${ }^{1}$ C. Briand, ${ }^{1}$ B. Cecconi, ${ }^{2}$ S. Célestin, ${ }^{15}$ D. Charrier, ${ }^{9}$ E. Chassande-Mottin, ${ }^{3}$ A. Coffre, ${ }^{2}$ I. Cognard, ${ }^{16,17}$ F. Combes,,${ }^{4,18}$ S. Corbel, ${ }^{19} \mathrm{C}$. Courte, ${ }^{20}$ A. Dabbech, ${ }^{1}$ S. Daiboo, ${ }^{15}$ R. Dallier, ${ }^{3}$ C. Dumez-Viou, ${ }^{21}$ M. N. El Korso, ${ }^{22}$ E. Falgarone, ${ }^{5}$ I. Falkovych,${ }^{20} \mathrm{~A}$. Ferrari, ${ }^{20} \mathrm{C}$. Ferrari, ${ }^{11} \mathrm{~K}$. Ferrière, ${ }^{20} \mathrm{C}$. Fevotte, ${ }^{22}$ A. Fialkov, ${ }^{23}$ M. Fullekrug, ${ }^{24}$ E. Gérard, J.-M. ${ }^{2}$ Grießmeier, ${ }^{25}$ B. Guiderdoni, ${ }^{2}$ L. Guillemot, ${ }^{26}$ J. Hessels, ${ }^{27}$ L. Koopmans, ${ }^{26}$ V. Kondratiev, ${ }^{1}$ L. Lamy, ${ }^{20}$ T. Lanz, ${ }^{28}$ P. Larzabal, ${ }^{29}$ M. Lehnert, ${ }^{22}$ F. Levrier, ${ }^{4,18}$ A. Loh, ${ }^{20}$ G. Macario, ${ }^{30}$ J.-J. Maintoux, ${ }^{15}$ L. Martin, ${ }^{20}$ D. Mary, ${ }^{1}$ S. Masson, ${ }^{31}$ M.-A. Miville-Deschenes, ${ }^{32}$ D. Oberoi, ${ }^{33}$ M. Panchenko, ${ }^{25}$ M. Pandey-Pommier, ${ }^{9}$ A. Petiteau, ${ }^{2}$ J.-L. Pinçon, ${ }^{15}$ B. Revenu, ${ }^{30}$ F. Rible, ${ }^{20} \mathrm{C}$. Richard, ${ }^{33}$ H. O. Rucker, ${ }^{16} \mathrm{P}$. Salomé, ${ }^{16} \mathrm{~B}$. Semelin, ${ }^{34} \mathrm{M}$. Serylak, ${ }^{6}$ O. Smirnov, ${ }^{35} \mathrm{~B}$. Stappers, ${ }^{3} \mathrm{C}$. Taffoureau, ${ }^{24} \mathrm{C}$. Tasse, ${ }^{2} \mathrm{G}$. Theureau, ${ }^{5} \mathrm{P}$. Tokarsky, ${ }^{3}$ S. Torchinsky, ${ }^{5}$ O. Ulyanov, ${ }^{24} \mathrm{~W}$. van Driel, ${ }^{1,5} \mathrm{I}$. Vasylieva, ${ }^{36} \mathrm{~J}$. Vaubaillon, ${ }^{10} \mathrm{~F}$. Vazza, ${ }^{24}$ S. Vergani, ${ }^{37}$ M. Was, ${ }^{38}$ R. Weber, and ${ }^{5}$ V. Zakharenko

\author{
${ }^{1}$ LESIA, Meudon, France, E-mail: philippe.zarka@obspm.fr \\ ${ }^{2}$ LPC2E, Orléans, France, ${ }^{3}$ USN, Nançay, France, ${ }^{4} \mathrm{CEA} / \mathrm{AIM}$, Saclay, France, ${ }^{5} \mathrm{RI}$ \\ NASU, Kharkiv, Ukraine, ${ }^{6}$ Univ. Cape, South Africa, ${ }^{7} \mathrm{CEA}$, Saclay, France, \\ ${ }^{8}$ ONERA, France, ${ }^{9}$ APC, Paris, France, ${ }^{10}$ Hamburg Observatory, Germany, ${ }^{11}$ IRAP, \\ Toulouse, France, ${ }^{12}$ LAM, Marseille, France, ${ }^{13}$ L2S, Nice, France, ${ }^{14}$ INAF, Italy, \\ ${ }^{15}$ Subatech, Nantes, France, ${ }^{16}$ LERMA, Paris, France, ${ }^{17}$ Collège de France, Paris, \\ France, ${ }^{18}$ Univ. Paris-Diderot, France, ${ }^{19}$ ESBAT, Tours, France, ${ }^{20}$ Lagrange OCA, \\ Nice, France, ${ }^{21}$ LEME Univ. Paris Ouest, France, ${ }^{22}$ ENS, Paris, France, ${ }^{23}$ Univ. Bath, \\ UK, ${ }^{24}$ GEPI, Meudon, France, ${ }^{25}$ CRAL, Lyon, France, ${ }^{26}$ ASTRON, The Netherlands, \\ ${ }^{27}$ Kapteyn Univ., The Netherlands, ${ }^{28}$ ENS, Cachan, France, ${ }^{29}$ IAP, Paris, France, \\ ${ }^{30}$ RETRAM, France, ${ }^{31}$ IAS, Orsay, France, ${ }^{32}$ MIT, USA, ${ }^{33}$ SRI, Graz, Austria, \\ ${ }^{34}$ Univ. Oxford, UK, ${ }^{35}$ Univ. Manchester, UK, ${ }^{36}$ IMCCE, Paris, France, ${ }^{37}$ LAPP, \\ Annecy, France, ${ }^{38}$ PRISME Polytech, Orléans, France
}

\begin{abstract}
NenuFAR is both a giant extension of the LOFAR and a large standalone instrument in the low-frequency range (10-85 MHz). It was designed in Nançay with national and international collaboration. Antenna radiators were modeled on the LWA antenna design whereas preamplifiers were designed in France. Antennas will be distributed in 96 mini-arrays of 19 dual-polarized elements, densely covering a disk of $400 \mathrm{~m}$ in diameter. A few mini-arrays are expected to lie at distances of 2-3 $\mathrm{km}$. A silent control-command system was designed, and the computer dialog with LOFAR defined. Receivers will include the LOFAR backend, a local beamformer and a local correlator. NenuFAR is in construction in Nançay and it was recently granted by the SKA office the official label of SKA pathfinder. Its exploitation will expand the scope of LOFAR scientific studies as well as permit new studies, preparing for SKA science. The NenuFAR concept has many points in common with GURT (the Giant Ukrainian Radio Telescope), with which it shares some technical studies, an its exploitation will benefit from a coordination with UTR-2. We describe the instrument, technical developments and science case.
\end{abstract}

Keywords: Radio astronomy; radio telescope; antenna array; antenna effective area.

\section{INTRODUCTION}

LOFAR is the new European multi-scale lowfrequency (LF) radio interferometer in the range of
$30-250 \mathrm{MHz}$ with baselines of from $\sim 50 \mathrm{~m}$ to $\sim 1000 \mathrm{~km}[1]$. The constitutive elements of LOFAR are phased arrays or "stations" distributed in the Netherlands and surrounding European countries. One 
of these stations, FR606, is installed in the Nançay radio observatory (France). Each station consists of two arrays of antennas and a "back-end" that preprocesses antenna signals (filtering, digitization, spectral channelization and beamforming). Preprocessed digital data are then sent at $\sim 3 \mathrm{Gbits} / \mathrm{sec}$ to the central computer (in Groningen, NL) that performs the correlations per interferometric baseline and/or final pencil beamforming. The Low-Band Antenna (LBA) array covers the range of $30-80 \mathrm{MHz}$ and consists of 96 elementary crossed dipoles in international stations such as FR606 (48 in Dutch stations). The High-Band Antenna (HBA) array covers the range of 110-250 $\mathrm{MHz}$ and consists of 96 "tiles" of 16 analog-phased crossed dipoles $(2 \times 24$ in Dutch stations). At any given time, the backend can be connected to either the LBA or the HBA (not both simultaneously). A third input to the backend exists, that was initially planned for an LBL (Low-Band Low, 10-50 MHz) array that never existed due to limited funding.

NenuFAR (formerly "LSS" for "LOFAR Super Station") exploits the availability of the third analog input of the LOFAR back-end to form a new LF phased array interferometer. No a priori constraints exist on its design and it will be fully compatible with LOFAR operations in the LBA band (i.e. that can be correlated with LBA arrays of other LOFAR stations, instead of the FR606 LBA array) and at the same time provide a considerably increased instantaneous sensitivity and frequency coverage.

\section{TeChNiCAL DESCRIPTION OF NENUFAR}

NenuFAR possesses a hierarchical design inspired of the HBA antenna field but adapted to a lower frequency band, which extend the LBA band (e.g. 10$90 \mathrm{MHz}$ ). The NenuFAR "tile" is composed of adapted dual-polarization antennas which signal is locally combined as a small phased array. Each tile provides a large gain from $10-15 \mathrm{MHz}$ to $85-87 \mathrm{MHz}$ (i.e. a ratio $f_{\max } / f_{\min }$ double of that of the LBA range). Their output is a pair (for the two linear polarizations) of analog signals that enters either the LBL analog inputs on the FR606 back-end or the analog input of a dedicated back-end installed close-by. The three main levels of the instrument drove three different design studies:

- The design of the antenna (feed and preamplifier)

- The distribution inside the LF "tile" or "miniarray" (the number, distribution of antennas and the phasing system)

- The distribution of these mini-arrays on the Nançay field (optimal for phased-array or interferometer performance vs. site constraints).

A lot of efforts has also been put in the design of a prototype receiver for the three first prototypes of mini-arrays.

\subsection{ANTENNA AND PREAMPLIFIER}

The diagram of the antenna radiator was optimized via electromagnetic simulations with the NEC code and validated with tests on the sky; optimized parameters include a broad and smooth beam (nearly isotropic, albeit with extinction below $20^{\circ}$ elevation) and maximum efficiency (related to electrical and ground losses), over a large frequency bandwidth; this implies an antenna radiation resistance and (low) reactance as constant as possible over the band of interest and versus time; cost effectiveness and compatibility with LOFAR strongly favored linearly polarized crossed dipoles; the optimal compromise is a "thick" inverted$\mathrm{V}$ dipole similar to the LWA Fork (see Fig.1), with a metallic ground screen [2].

Three designs have been studied and realized for the antenna preamplifier, which is a key element for the antenna gain and its susceptibility to radiofrequency interference (RFI): (1) the "GURT2" design from the Institute of Radio Astronomy, Kharkiv, Ukraine, (2) the Subatech/Nançay design, and (3) the Nançay microelectronics laboratory design. (1) is based on discrete components, whereas (2) \& (3) are based on ASIC circuits. All have good characteristics, with a noise $\sim 10 \mathrm{~dB}$ below the sky noise level. For NenuFAR-1, the Subatech/Nançay design was selected.

\subsection{THE MINI-ARRAY}

The number of antennas within each tile should be of the order of 16 (again as in HBA tiles) in order to provide at least an order of magnitude increase of the instantaneous sensitivity. The antenna distribution within each LF tile was optimized for a low side lobe level and a large field of view symmetrical around the zenith; in [3] it is found that a distribution with a central antenna surrounded by two circular rings of antennas meets these requirements, especially if the rings have different (or no) symmetry axes, i.e. if the global distribution cannot be superposed to itself by a rotation $<2 \pi$. In parallel, we have calculated that analog phasing of each LF tile using 7-bit delay lines (cable lengths) allows to perform achromatic phasing over the whole NenuFAR band (10-87 MHz) with gain variations $<10 \%$ across the beam, and provide one input per polarization to the backend. In order to be cost-effective, delay lines must be mutualized for groups of antennas, e.g. by arranging antennas with a regular spacing in two orthogonal directions. Taking into account this constraint we modified the above optimized antenna distributions to obtain an LF Miniarray LSS tile of 19 antennas (a central one surrounded by an hexagon of 6 antennas and a second 


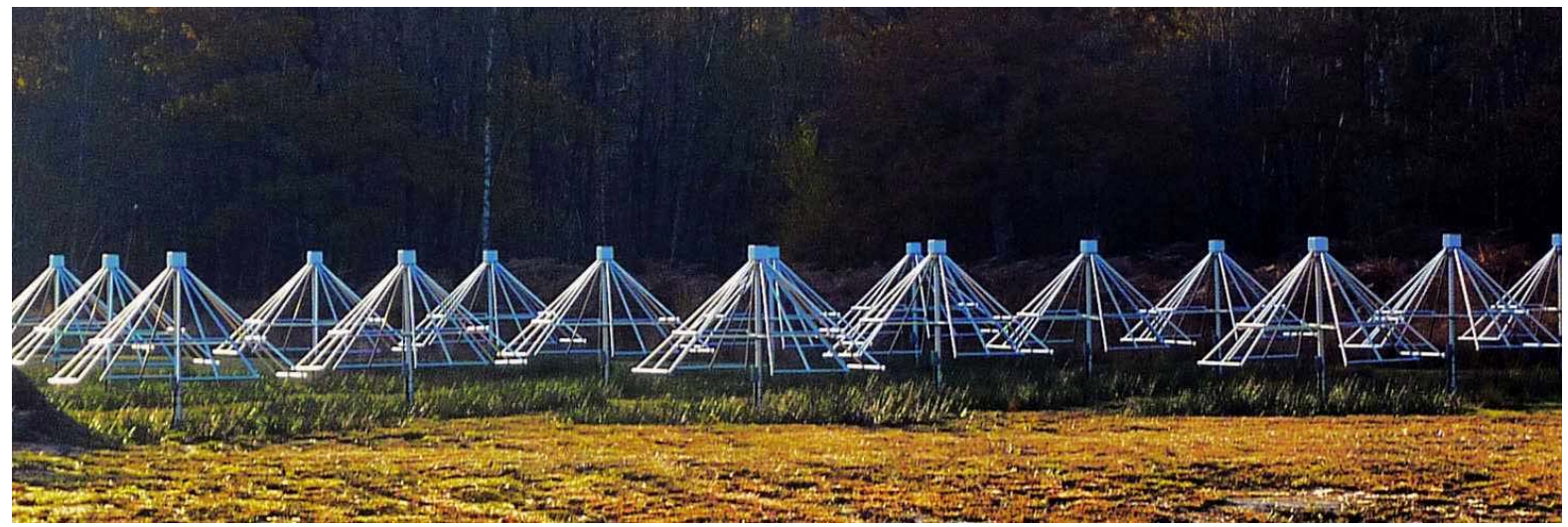

Fig. 1. Picture of the prototype mini-array.

one of 12 antennas, or equivalently regular lines of $3 /$ 4 / 5 / 4 / 3 antennas with each line shifted by $1 / 2$ inter-antenna spacing relative to its neighbors (Fig. 2). The absolute value of inter-antenna spacing was set to $5.5 \mathrm{~m}$ in order to maximize the effective area without overlap at LF, while keeping the NenuFAR extent compatible with its hosting at the Nançay station. The instantaneous LF tile beam will have an angular size of $10^{\circ}$ to $50^{\circ}$ over the NenuFAR spectral range $\left(\sim 25^{\circ}\right.$ at $30 \mathrm{MHz}$ ).

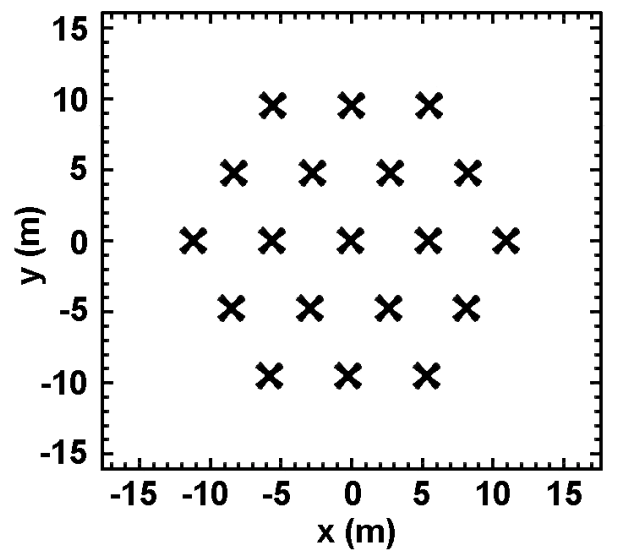

Fig. 2. Schematic layout of the mini-array

\subsection{NENUFAR AS AN INTERFEROMETER / PHASED ARRAY}

The 96 tiles should be arranged in a relatively dense layout (within a few hundred meters diameter), providing a smooth overall beam with a low side lobe level and compatible with the available land in an observatory such as the Nançay station, and at the same time minimize the overlap between antennas effective areas in order to maximize the NenuFAR sensitivity.

The optimal distribution of the 96 LF tiles was computed using the algorithm [4], taking into account a "site mask" of the Nançay station including its limits and forbidden areas (the station FR606 itself and other antennas of the site). It provides a smooth, Gaussian distribution of visibilities in the $(\mathrm{u}, \mathrm{v})$ plane, and thus a near-Gaussian NenuFAR beam pattern. The layout of trenches and cables connecting the LF tiles to the FR606 backend was optimized using a reasonable cost ratio per unit length of trench/cable in input to a specific optimization algorithm [5]. The obtained LF tiles distribution and layout is displayed in Fig.3. It implies a NenuFAR beam size of $0.5^{\circ}-3^{\circ}$ $\left(\sim 1.5^{\circ}\right.$ at $\left.30 \mathrm{MHz}\right)$. In order to reduce the side lobe level resulting from the regular antennas arrangement in the LF tiles, each tile will be rotated by a random amount with respect to each other, but all crossed dipoles within all tiles will be oriented along the same directions, at $45^{\circ}$ from the meridian.

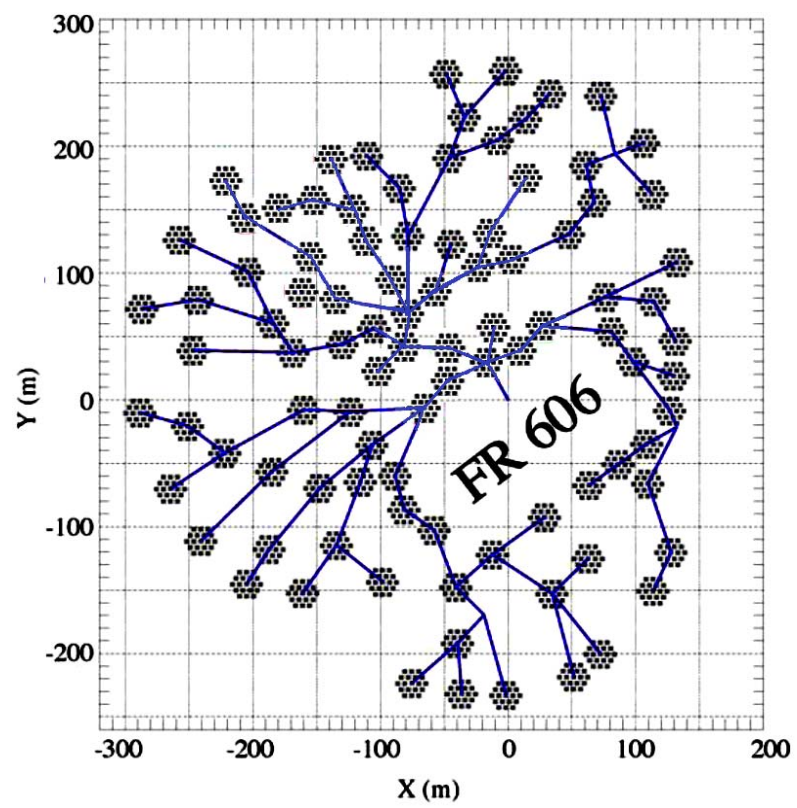

Fig. 3. Distribution of the 96 mini-arrays around the LOFAR station FR606 in Nançay. 


\section{NENUFAR PERFORMANCE AND OBSERVING MODE}

\subsection{OBSERVING CAPABILITIES AND IMPROVEMENTS BROUGHT BY NENUFAR}

NenuFAR by itself, is not a powerful imaging instrument, but its collecting area, and thus its sensitivity, will bring several significant improvements to LOFAR:

- The long LOFAR baselines including the NenuFAR will be $\sim 19$ times more sensitive than long baselines between two LBA arrays; as the available radio power corresponding to one angular details is generally weak, this increased sensitivity will give access to an increased number of calibrators (typically $\sim 10$ ) in the vicinity of the studied target; this will improve LOFAR's capabilities for high resolution imaging in the LBA range.

- By adding $96 \times 19=1824$ antennas to the present 2700 LOFAR LBA antennas, the NenuFAR will almost double LOFAR's sensitivity in the LBA imaging mode.

- When most of the "core" (closely-packed) LOFAR stations will be used for time-demanding projects such as observing the Epoch of Reionization (EoR), the NenuFAR will provide an alternative core and, by correlation with the "remote" LBA arrays, will preserve good imaging capability in parallel with the above projects; provided that the central correlator can handle two streams of data from two LOFAR sub-arrays (namely the core, and the NenuFAR + remote stations) the NenuFAR will improve LOFAR-LBA imaging capabilities for a significant fraction of the time.

- Correlation of signals from the LF tiles within the NenuFAR will provide sensitive baselines 2 to 3 times shorter than a LOFAR station diameter - the shortest baseline presently available, permitting to image large-scale structures, larger than an instantaneous station beam $\left(\sim 10^{\circ}\right.$ at $\left.30 \mathrm{MHz}\right)$; short baselines are presently available by correlation of LBA antennas within a station, but with much lower sensitivity.

- The NenuFAR will also be a very large standalone instrument: it will have an effective area (and thus sensitivity) $\sim 19$ times larger than the LBA array of an international station, i.e. $<70 \%$ to $85 \%$ of all LOFAR-LBA arrays, but this area will be instantaneously and fully available during use, especially in coherent tied-array (or phased-array) beam mode (TAB); by contrast, the coherent phasing of LOFAR-LBA array signals is limited to the 24 core stations that share the same reference clock (and have the same ionosphere above them); thus, the instantaneous sensitivity of the NenuFAR in coherent TAB mode will be 1.6 times better than the LOFAR-LBA one (Fig.4).
- Finally, in standalone mode, the NenuFAR will extend the observation bandwidth to significantly lower frequencies than the LBA range.

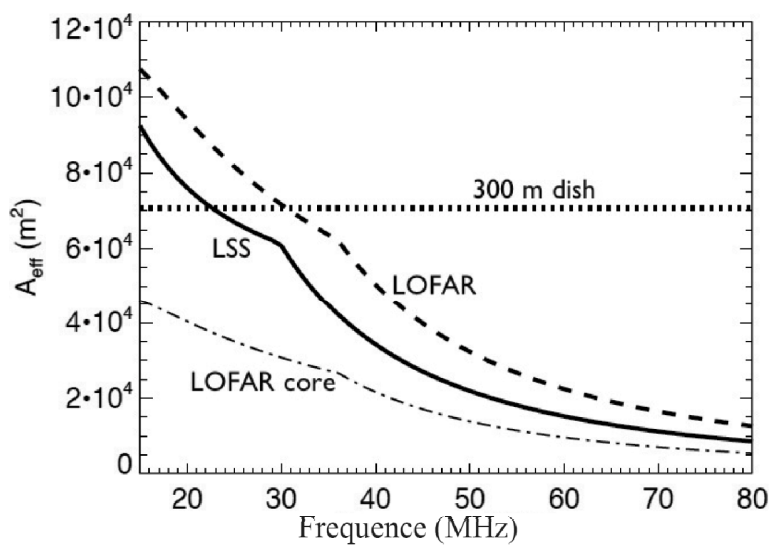

(a)

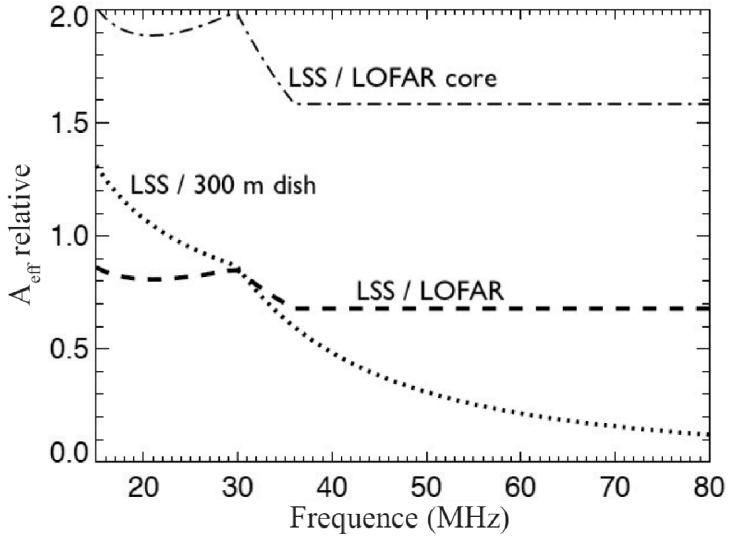

(b)

Fig. 4. Theoretical absolute (a) and relative (b) effective collecting areas of NenuFAR between 15 and $80 \mathrm{MHz}$ for NenuFAR (NenuFAR), the full LOFARLBA, the LOFAR core only and a typical $300 \mathrm{~m}$ diameter dish radio telescope (Arecibo).

The NenuFAR pointing will be controlled by a dedicated LCU (Local Command Unit) connected to 96 electronic modules, one in each LF tile, specifying the phasing scheme to be applied at any given time (different for each LF tile due to their random rotations); these modules are designed to be completely "radio-quiet" outside pointing time; pointing will occur at intervals from 20 to $60 \mathrm{sec}$, ensuring low gain variation in the main beam direction. The NenuFAR LCU will be connected to the LOFAR LCU of station FR606. When NenuFAR will be used jointly with other LOFAR LBA arrays (so-called "International" mode), pointing orders will come from the LOFAR operations center via the LOFAR LCU and be translated into LF tiles phasing commands by the NenuFAR LCU; data recorded by the LOFAR backend will be sent to the LOFAR central correlator. In "Standalone" mode, pointing orders will come from a local command computer, and the data will be recorded locally. 


\subsection{STANDALONE Use With DedicAted RECEIVER}

The standard NenuFAR concept consists of the 96 additional LF tiles and their phasing and command system, that are connected to the LOFAR backend of FR606. The contract of any European station owner with the International LOFAR Telescope board includes the right to use that station in standalone mode for $\sim 10 \%$ of the time. As the standard LOFAR station backend only allows to record low frequency-time resolutions observations (typically in $200 \mathrm{kHz}$ in $1 \mathrm{sec}$ bins), the full scientific exploitation of standalone NenuFAR observations requires either LOFAR's "Single Station" mode or a dedicated post-backend [6]. The former consists of a sub-array formed by a single station, which high-resolution data are sent to the central correlator for processing as TAB data. The latter is for example the ARTEMIS (Advanced Radio Transient Event Monitor and Identification System) post-backend dedicated to transients detection and study, that ingests high resolution station data and locally computes high-resolution time-frequency planes including parametric dedispersion [7].

If one wants to compute locally more than timefrequency planes with NenuFAR standalone data (e.g. auto- and cross-correlations of tile signals), then a dedicated receiver is necessary. Such a dedicated receiver has been studied in the frame of the NenuFAR design study. It will consist of either a post-LOFARbackend (ARTEMIS-like), or a fully independent backend. The LOFAR backend digitizes the LBA or NenuFAR tile signals, channelizes it in $200 \mathrm{kHz}$ bands (called "subbands"), and computes beamforming within each subband. The beamformed signal of 244 subbands is sent to the LOFAR central correlator. The latter further channelizes (down to $0.76 \mathrm{kHz}$ resolution) the signals of all subbands from all stations, and computes auto-/cross-correlations and/or incoherent or coherent (adequately time-shifted) summation in order to produced polarized images and/or TAB data.

A post-LOFAR-backend dedicated receiver would ingest locally the station products (as does ARTEMIS) and channelize, auto-/cross-correlate, and integrate them. A fully independent backend would perform the tasks of both the LOFAR backend and the central correlator, but in an optimized integrated way. A large computing power is required, but a preliminary design study suggests that this is within the scope of new generation FPGAs. Additional "intelligent" processing like RFI mitigation or parametric dedispersion could be included. Transient Buffer Board data will also be processed in all cases. A dedicated receiver is important not only because it allows local, flexible processing, but primarily because it will greatly increase the duty-cycle of the NenuFAR standalone use beyond the "guaranteed" $10 \%$ fraction of the time, albeit with some pointing constraints. With a post-LOFAR-backend receiver, usable in parallel to all NenuFAR observations (standalone or in correla- tion with LOFAR LBA), the standalone analysis of NenuFAR data will be possible $100 \%$ of the time during which the NenuFAR tiles are connected to the FR606 backend, but the target will necessarily be within the $0.5^{\circ}-3^{\circ}$ NenuFAR beam fixed by the current LOFAR observation program. Conversely, with a fully independent backend that would process NenuFAR data in parallel with the standard station backend, standalone mode becomes possible $100 \%$ of the time, whatever array (LBA, HBA or NenuFAR) is connected to the LOFAR backend. The only pointing constraint of this standalone NenuFAR mode is that the target must be located within the $10^{\circ}-50^{\circ}$ analog LF tile beam.

\section{NenUFAR PERFormanCE AND OBSERVING Mode}

The final distribution of the 96 mini-arrays was set by the positioning constraints around the FR606 station and the power distribution over the entire field. NenuFAR was divided in "petals" allowing a modular and flexible implantation of the instrument. Starting from the three prototype mini-arrays that were built during the design study, $\sim 1 / 4$ of the instrument (in fact 26 mini-arrays, defined as "NenuFAR-1") is planned to be built before early 2015 . The chosen mini-arrays provide a decent instrument PSF that will improve gradually with the construction of additional petals of the array while keeping the best azimuthal symmetry of the mini-array distribution (and therefore, the azimuthal symmetry of the instrumental beam and interferometric coverage).

The expected performance of NenuFAR is compared to that of LOFAR in Fig.4 and a summary table of the instrumental characteristics, compared to other classical radio instrument can be found in Table 1 . The expected sensitivities (for a 8-hour time integration over the $\sim 10-90 \mathrm{MHz}$ ) are $120-240 \mathrm{mJy}$ for NenuFAR-1, 50-100 mJy for the whole NenuFAR.

\section{Conclusion}

The NenuFAR characteristics are compared to those of other existing international instruments in Table 1. In the European context, several instrumental projects are developed by LOFAR participants, such as ARTEMIS (from Oxford Univ., see above) or AARTFAAC (from Univ. Amsterdam - it aims at cross-correlating the 288 LBA and HBA signals from the 6 central LOFAR stations to perform permanent all-sky monitoring [8]).

NenuFAR is a LOFAR extension and a standalone instrument with emphasis on very high instantaneous sensitivity. We foresee it as an "Arecibo in Nançay". It is also a SKA precursor for the French community, both scientific and technical (for SKA-low).

It is interesting also the operation of NenuFAR for the Universe studies in coordination with another ground-based and space low frequency instruments such as UTR-2, URAN, GURT, NDA, NRT, LOFAR, 
Table. 1. Characteristics of NenuFAR and NenuFAR-1 compared to those of large LF radio instruments (observing below $100 \mathrm{MHz}$ ) existing or in project. (a) at $20 \mathrm{MHz}$, (b) at $30 \mathrm{MHz}$, (c) at $150 \mathrm{MHz}$.

\begin{tabular}{|c|c|c|c|c|c|c|}
\hline Name & $\mathrm{N}_{\text {antennas }}$ & $\begin{array}{c}\text { Effective } \\
\text { area, } \\
\left(\mathrm{m}^{2}\right)\end{array}$ & $\begin{array}{c}\text { Frequency } \\
\text { range } \\
(\mathrm{MHz})\end{array}$ & $\begin{array}{c}\text { Angle } \\
\text { resolution } \\
\text { (degrees) }\end{array}$ & $\mathrm{N}_{\text {beams }}$ & $\begin{array}{c}\text { Polarization } \\
\text { type }\end{array}$ \\
\hline NDA & 144 & $4000^{(a)}$ & $10-110$ & $11^{\mathrm{o}}(\mathrm{a})$ & $1 b$ & 2 circ \\
\hline UTR-2 & 2040 & 143000 & $8-32$ & $0.5^{\mathrm{o}}$ & $5 b$ & $1 \operatorname{lin}$ \\
\hline VLA & 27 & $\sim 2000$ & $73-74.5$ & $0.5^{\prime}$ & $1 b$ & 2 circ \\
\hline LWA & 256 & $8000^{(a)}$ & $10-88$ & $9^{o(a)}$ & $\begin{array}{l}4 \mathrm{~b} \times 20 \\
\mathrm{MHz}\end{array}$ & $2 \operatorname{lin}$ \\
\hline MWA & 2048 & $\sim 2000^{(\mathrm{c})}$ & $80-300$ & $3^{\prime(c)}$ & $\begin{array}{l}1 \mathrm{bx} 30 \\
\mathrm{MHz}\end{array}$ & $2 \operatorname{lin}$ \\
\hline $\begin{array}{l}\text { LOFAR- } \\
\text { LBA }\end{array}$ & 2688 & 72000 & $30-80$ & $2^{\prime \prime(b)}$ & $\begin{array}{c}8 \mathrm{~b}+\mathrm{nx} 4 \\
\mathrm{MHz}\end{array}$ & $2 \operatorname{lin}$ \\
\hline NenuFAR-1 & 494 & $\sim 17000^{(\mathrm{b})}$ & $15-80$ & $\sim 3^{\circ}(\mathrm{b})$ & $\begin{array}{l}4 \mathrm{bx} 65 \\
\mathrm{MHz}\end{array}$ & $2 \operatorname{lin}$ \\
\hline $\begin{array}{l}\text { NenuFAR } \\
\text { Standalone }\end{array}$ & 1824 & $62000^{(b)}$ & $15-80$ & $1.5^{\mathrm{o}}(\mathrm{b})$ & $\begin{array}{c}4 \mathrm{bx} 65 \\
\mathrm{MHz}\end{array}$ & $2 \operatorname{lin}$ \\
\hline $\begin{array}{l}\text { NenuFAR } \\
+ \text { LOFAR }\end{array}$ & 4512 & $134000^{(b)}$ & $30-80$ & $2^{\prime \prime(b)}$ & $\begin{array}{c}8 \mathrm{~b}+\mathrm{nx} 4 \\
\mathrm{MHz}\end{array}$ & $2 \operatorname{lin}$ \\
\hline SKA & $>3000$ & 1000000 & $70-10000$ & $<0.1^{\prime \prime}$ & Many & $2 \operatorname{lin}+\operatorname{circ}$ \\
\hline
\end{tabular}

SKA, LWA, Cassini, STEREO, FERMI, JUNO, HST, Farside Explorer, etc.

\section{REFERENCES}

1. Van Haarlem, M.P., Wise M.W., Gunst A.W. et al. 2013, 'LOFAR: The Low Frequency ARray', Astron. and Astrophys. 556,

2. Girard, J. N., Zarka, P., Tagger, M. et al. 2011, 'Antenna Design and Distribution for a LOFAR Super Station in Nançay' In: Planetary Radio Emissions VII. Austrian Acad. Sci. Press, Vienna. Ed. H. O. Rucker et al. 495-503.

3. Girard, J. N., Zarka, P., Tagger, M. et al. 2012, 'Antenna design and distribution of the LOFAR Super Station', C.R. Phys., 13, 33-37.

4. Boone, F. 2001, 'Interferometric array design: Optimizing the locations of the antenna pads' $A s$ tron. and Astrophys. 377, 368-376.
5. Vasko, F. J., Barbieri, R. S., Rieksts, B. Q., Reitmeyer, K. L., and Stott Jr., K. L. 2002, 'The cable trench problem: combining the shortest path and minimum spanning tree problems' Computers and Operations Res., 29, 441-458.

6. Serylak, M., Karastergiou, A., Williams, C. et al. 2012, 'Observations of Transients and Pulsars with LOFAR International Stations', Proc. Electromagnetic Radiation from Pulsars and Magnetars Conf., Zielona Gora, Poland, 83-86.

7. Armour, W., Karastergiou, A., Giles, M. et al. 2011, 'A GPU-based Survey for Millisecond Radio Transients Using ARTEMIS', Proc. ADASS XXI. Ed. P. Ballester and D. Egret. 33-37.

8. Prasad, P. and Wijnholds, S. J. 2012, 'AARTFAAC: Towards a 24x7, All-sky Monitor for LOFAR', Proc. New Windows on Transients across the Universe, London, UK. 